\title{
Polarization of radiation scattered in magnetized turbulent envelopes
}

\author{
N. A. Silant'ev ${ }^{1,2}$ \\ 1 Instituto Nacional de Astrofísica, Óptica y Electrónica, Apartado Postal 51 y 216, CP 72000, Pue. México \\ e-mail: silant@inaoep.mx \\ 2 Main Astronomical Observatory of the Russian Academy of Sciences, Pulkovo, St.Petersburg 196140, Russia \\ Received 29 April 2005 / Accepted 25 November 2005
}

ABSTRACT

We consider the single scattering of the non-polarized radiation of a central star on the electrons of a plasma circumstellar envelope. We assume that such an envelope is magnetized and consists of a turbulent plasma. Two types of envelopes are considered: a spherical envelope with the dipole magnetic field and a cone-like jet with the radial magnetic field. It is shown that the existence of turbulent motions in the envelopes drastically changes the polarization degree spectrum $p(\lambda)$ and the positional angle spectrum $\chi(\lambda)$ of scattered radiation compared to the nonturbulent case. The difference in spectra can be used to estimate the ratio for the energy of magnetic fluctuations to the energy of a regular magnetic field.

Key words. polarization - magnetic fields

\section{Introduction}

There are a lot of observational data on the existence of stellar winds, jets, and envelopes for various stars, quasars, and active galactic nuclei (see for example, Hartmann 1983; Impey et al. 1991; Landstreet 1992; Wills et al. 1992; Paatz \& Camenzind 1996). Many of these envelopes possess magnetic fields (see recent observations of Udompraset et al. 1997; Ishwara-Chandra et al. 1998; Pentericci et al. 2000). Theoretical models of objects with envelopes, jets, and accretion disks widely use the concepts of magneto hydrodynamics (see the review of Blaes 2003), i.e. they have to be considered as magnetized cosmic objects. Spectrophotometric observations are used to confirm or reject the theoretical models of these complex cosmic objects (Cherepashchuk 2001).

Integral polarization of radiation from point-like cosmic objects (the intrinsic polarization) arises if the structure of these objects is not axially symmetric with respect to the line of sight. Generally, this asymmetry implies the asymmetry of physical conditions in the cosmic objects and their nearest vicinity, not only the asymmetry of their geometric structure. Thus, the spherical envelope with a dipole magnetic field is not an axially symmetric object, and it can produce non-zero integral linear polarization due to different Faraday rotations of the polarization plane of radiation scattered in various volumes of the envelope.

There are a lot of polarimetric observations of various cosmic objects. For example, Lamy \& Hutsemekers (2000) present the optical polarization of 47 quasi-stellar objects. Most QSOs in this catalogue have rather small intrinsic polarization - only 10 objects have $p>1 \%$. The maximum polarization $\cong 6 \%$ has the object B1333+2840. Schmidt \& Hines (1999) give the polarization of broad absorption lines for 53 QSOs, where only 15 objects show large $(p>3 \%)$ polarization. The catalogue of optical polarization of 83 QSOs (Wills et al. 1992) shows that the fraction of highly polarized $(p>3 \%)$ objects is smaller for more distant $(z>1)$ QSOs. The possible explanation of this is given in Gnedin \& Silant'ev (2002). The polarimetry of a number of active galaxies is presented in Young et al. (1996). There are many polarimeric observations of supernova explosions (see Leonard et al. 2000). The observed magnitude of the polarization varies from $0.2 \%$ up to $2 \%$ and is explained as being due to Thomson scattering in aspherical envelopes. We also note the X-ray polarimetric observations of ScoX-1 (Long et al. 1979) and CygX-1 (Long et al. 1980). For ScoX-1 they find $p(2.6 \mathrm{keV}) \cong 0.4 \%$ and $p(5.2 \mathrm{keV}) \cong 1.31 \%$. The CygX-1 gives larger polarization $-p(2.6 \mathrm{keV}) \cong 2.4 \%$ and $p(5.2 \mathrm{keV}) \cong 5.3 \%$. Now the gamma-ray polarimetry is arising. It should be stressed that optical polarimetry can measure very small polarization $\sim 0.01 \%$ (see catalogues mentioned above). We do not mention here the numerous polarimetric observations of the usual stars. Some of them can be magnetized and possess magnetized eruptive envelopes (see Chesneau et al. 2003).

This paper deals with the integral polarization that arises due to the existence of Faraday rotation of the polarization plane of the radiation, scattered in various magnetized envelopes. The partly polarized scattered radiation undergoes different Faraday rotations for various scattering volumes in an envelope. As a result, the integral scattered radiation from the envelope acquires non-zero net polarization even if 
the envelope is axially symmetric geometrically with respect to the line of sight. The angle of Faraday rotation $\psi \propto \lambda^{2}$ depends on the wavelength of the radiation. This gives rise to the peaklike form of the polarization degree spectrum $p(\lambda)$ (see Gnedin \& Silant'ev 1980, 1984, 1997 and Gnedin et al. 2005). The particular form of the $\lambda$-dependence is different for various models of the electron number density, which gives the opportunity to estimate both the magnetic field value and the electron density distribution. This new technique is available if the spectra of polarization are observed; yet in the papers mentioned above, only the regular magnetic field is considered.

It should be noted that the known Zeeman effect technique for estimating the magnetic field in a vicinity of the objects with envelopes is frequently invalid due to the absence of suitable spectral lines. Thus, the Faraday rotation mechanism for polarizing of the integral radiation is a very important additional technique for estimating the existing magnetic fields.

The goal of this paper is to investigate how the spectrum $p(\lambda)$ and the positional angle spectrum $\chi(\lambda)$ in turbulent envelopes change when the magnetic field acquires the fluctuating component. First, we review the mechanism of polarization in non-turbulent magnetized envelopes. Then, we proceed by considering turbulent envelopes in Sect. 3. In Sects. 4 and 5 the spherical and cone-like envelopes are considered. Section 6 deals with the asymptotical behavior of polarization at large wavelengths.

\section{Polarization of radiation scattered in non-turbulent envelopes}

To start, we write the usual transfer equations for Stokes parameters $I(\boldsymbol{r}, \boldsymbol{n}), Q(\boldsymbol{r}, \boldsymbol{n})$, and $U(\boldsymbol{r}, \boldsymbol{n})$ that describe the intensity and linear polarization of radiation:

$$
\begin{aligned}
(\boldsymbol{n} \nabla) I(\boldsymbol{r}, \boldsymbol{n})= & -\alpha I(\boldsymbol{r}, \boldsymbol{n})+N_{\mathrm{e}} \sigma_{\mathrm{T}} B_{I}(\boldsymbol{r}, \boldsymbol{n}), \\
(\boldsymbol{n} \nabla) Q(\boldsymbol{r}, \boldsymbol{n})= & -\alpha Q(\boldsymbol{r}, \boldsymbol{n})-N_{\mathrm{e}}(\boldsymbol{r}) \sigma_{\mathrm{T}} \delta \cos \Theta U(\boldsymbol{r}, \boldsymbol{n}) \\
& +N_{\mathrm{e}} \sigma_{\mathrm{T}} B_{Q}(\boldsymbol{r}, \boldsymbol{n}) \\
(\boldsymbol{n} \nabla) U(\boldsymbol{r}, \boldsymbol{n})= & -\alpha U(\boldsymbol{r}, \boldsymbol{n})+N_{\mathrm{e}}(\boldsymbol{r}) \sigma_{\mathrm{T}} \delta \cos \Theta Q(\boldsymbol{r}, \boldsymbol{n}) \\
& +N_{\mathrm{e}} \sigma_{\mathrm{T}} B_{U}(\boldsymbol{r}, \boldsymbol{n}),
\end{aligned}
$$

where $\sigma_{\mathrm{T}}=8 \pi r_{\mathrm{e}}^{2} / 3$ is the Thomson cross-section, $r_{\mathrm{e}}=e^{2} / m_{\mathrm{e}} c^{2}$ is the classic radius of electron, $\alpha=N_{\mathrm{e}} \sigma_{\mathrm{T}}+k_{\mathrm{a}}$ the extinction factor, $k_{\mathrm{a}}$ true absorption coefficient, $N_{\mathrm{e}}(\boldsymbol{r})$ the electron number density, $\boldsymbol{n}$ the direction of the light propagation, $\Theta$ the angle between $\boldsymbol{n}$ and magnetic field $\boldsymbol{B}$. The $B_{I, Q, U}$-terms describe the scattering of radiation on free electrons (see for example, Chandrasekhar 1950). Dimensionless parameter $\delta$ characterizes the Faraday rotation effect:

$\delta=\frac{3}{8 \pi^{2}} \cdot \frac{c \omega_{B}}{r_{\mathrm{e}} v^{2}} \equiv \frac{3 \lambda}{4 \pi r_{\mathrm{e}}} \cdot \frac{\omega_{B}}{\omega} \cong 0.8 \lambda^{2}(\mu \mathrm{m}) B(\mathrm{G})$

where $\lambda$ and $v$ are the wavelength and frequency of the scattered light, respectively, and $\omega_{B}=e B / m_{\mathrm{e}} c$ is the cyclotron frequency.

The angle of Faraday rotation of the polarization plane is:

$$
\psi(\boldsymbol{n})=\frac{1}{2} \delta \tau_{\mathrm{T}} \cos \Theta
$$

where $\tau_{\mathrm{T}}=N_{\mathrm{e}} \sigma_{\mathrm{T}} s$ is the Thomson optical depth along a path $s$.
For an optically thin envelope, the Stokes parameters of the single-scattered radiation are determined by the expressions (Gnedin \& Silant'ev 1984, 1997; Gnedin et al. 2005):

$$
\begin{aligned}
F_{I}(\boldsymbol{n})= & \frac{L}{4 \pi R^{2}} \cdot \frac{3 \sigma_{\mathrm{T}}}{16 \pi} \int \mathrm{d} V \frac{N_{\mathrm{e}}(\boldsymbol{r})}{r^{2}}\left\{\frac{8 r^{2}}{3 R_{\mathrm{s}}^{2}}\left(1-\sqrt{1-\frac{R_{\mathrm{s}}^{2}}{r^{2}}}\right)\right. \\
& \left.+\left(-\frac{1}{3}+\cos ^{2} \vartheta\right) \sqrt{1-\frac{R_{\mathrm{s}}^{2}}{r^{2}}}\right\}, \\
F_{Q}(\boldsymbol{n})= & -\frac{L}{4 \pi R^{2}} \\
& \times \frac{3 \sigma_{\mathrm{T}}}{16 \pi} \int \mathrm{d} V \frac{N_{\mathrm{e}}(\boldsymbol{r})}{r^{2}} \sqrt{1-\frac{R_{\mathrm{s}}^{2}}{r^{2}}} \sin ^{2} \vartheta \cos 2(\varphi+\psi), \\
F_{U}(\boldsymbol{n})= & -\frac{L}{4 \pi R^{2}} \\
& \times \frac{3 \sigma_{\mathrm{T}}}{16 \pi} \int \mathrm{d} V \frac{N_{\mathrm{e}}(\boldsymbol{r})}{r^{2}} \sqrt{1-\frac{R_{\mathrm{s}}^{2}}{r^{2}}} \sin ^{2} \vartheta \sin 2(\varphi+\psi),
\end{aligned}
$$

where $L$ is the stellar spectral luminosity ( $\operatorname{erg~s}^{-1} \mathrm{~Hz}^{-1}$ ), $\vartheta$ and $\varphi$ are the polar and azimuthal angles of a radius-vector $\boldsymbol{r}$ in the reference frame with $z$-axis directed to the observer (along the line of sight $\boldsymbol{n}$ ), and $R$ is the distance to the observer. The integration is carried out over all visible volumes of an envelope. We choose the $X$-axis lying in the plane $(\boldsymbol{n h})$, where $\boldsymbol{h}$ is the direction of magnetic dipole $\boldsymbol{M}$ for a spherical magnetized envelope, or the direction of the axis of the cone-like envelope (see Fig. 1), $\psi \equiv \psi(\boldsymbol{n}, \boldsymbol{r})$ is the Faraday rotation angle corresponding to the propagation of the light beam scattered in the point $\boldsymbol{r}$. This angle can be calculated by path integration along the line of sight:

$\psi(\boldsymbol{n}, \boldsymbol{r})=0.4 \sigma_{\mathrm{T}} \lambda^{2}(\mu \mathrm{m}) \int \mathrm{d} l N_{\mathrm{e}}\left(\boldsymbol{r}^{\prime}\right) \boldsymbol{n} \cdot \boldsymbol{B}$.

The vector $\boldsymbol{r}^{\prime}$ runs along the line of sight $\boldsymbol{n}$. The coordinates $\boldsymbol{r}^{\prime}=\left(r^{\prime}, \vartheta^{\prime}, \varphi^{\prime}\right)$ are related to $\boldsymbol{r}=(r, \vartheta, \varphi): \varphi=\varphi^{\prime}$ and $r \sin \vartheta=r^{\prime} \sin \vartheta^{\prime}$, which gives the following expression for the path element: $\mathrm{d} l=r \mathrm{~d} \vartheta^{\prime} \sin \vartheta /\left(\sin \vartheta^{\prime}\right)^{2}$.

Near the surface of a star, the volume element $\mathrm{d} V$ is illuminated from the semi-sphere of directions, so the star does not look like a point source of radiation with the flux $L / 4 \pi r^{2}$. Dolginov et al. (1995) showed how to change the usual formulae to take this effect into account. Our formulae correspond to the case of the intensity of radiation escaping from the star surface element is isotropic $(I(\mu)=$ const.), where $\mu$ is the cosine of the angle between outward normal $N$ to the surface and the direction of the escaping. In Dolginov et al. (1995) the analytic formulae are given for the more realistic case $I(\mu)=A+B \mu$ accounting for the gradient of the Planck-function in an atmosphere.

The considered case $I(\mu)=A$ corresponds to homogeneous distribution of thermal sources in an atmosphere. It gives the lower limit for possible degree of polarization. If $r \gg R_{\mathrm{s}}$, formulae (4) transform to the case of a point-like star that corresponds to the upper limit for polarization. It is seen that the scattered radiation is non-polarized near the star surface, which means that the incident radiation falls isotropically from the 


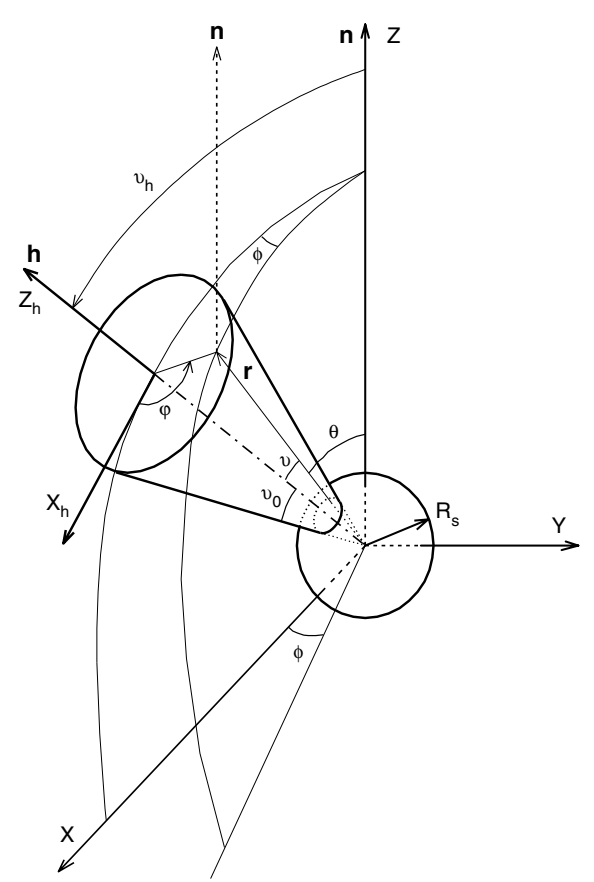

Fig. 1. The position of the cone-like envelope and the various angles used in the text. The dotted line presents the direction to an observer.

semi-sphere of directions. Thus, this effect diminishes (sometimes to $\sim 30 \%$ ) the net polarization compared with a pointlike star. This effect was first taken into account in Pogodin (1992). Usually one supposes that illuminating sources (for brevity we call them "stars") are in LTE-state and radiate the non-polarized light.

Formulae (4) for the Stokes parameters $F_{I}, F_{Q}$, and $F_{U}$ (erg cm $\mathrm{cm}^{-2} \mathrm{~s}^{-1} \mathrm{~Hz}^{-1}$ ) take only single-scattered radiation into account. In this approximation by calculation of the degree of polarization $p=\sqrt{F_{Q}^{2}+F_{U}^{2}} / F_{I}$ one can take the direct radiative flux from the star as the flux $F_{I}: F_{I}=L / 4 \pi R^{2}$. Sometimes one supposes that the direct radiation from the star is obscured by some dense matter (for example, the edge of an accretion disk). In this case the observed radiation flux is equal to $F_{I}$ from Eq. (4) and the integral polarization from the envelope will be much greater than that considered below.

Clearly, that the flux of single-scattered radiation is proportional to the optical depth of an envelope $\tau_{\text {env }}$ (the exception, see Sect. 5.1), means the parameters $F_{Q}(\boldsymbol{n})$ and $F_{U}(\boldsymbol{n})$ can be presented in the form:

$F_{Q, U}(\boldsymbol{n})=\frac{L}{4 \pi R^{2}} \tau_{\mathrm{env}} f_{Q, U}\left(\delta_{\mathrm{s}} \tau_{\mathrm{env}}, \vartheta_{h}, \vartheta_{0}\right)$,

where $\delta_{\mathrm{s}}=0.8 \lambda^{2}(\mu \mathrm{m}) B_{\mathrm{s}}(\mathrm{G})$ is parameter (2) at the star's surface, and $B_{\mathrm{s}}$ is the magnetic field on some point of this surface (particularly, $B_{\mathrm{s}}=M / R_{\mathrm{s}}^{3}$ for the dipole magnetic field). The angles $\vartheta_{h}$ are the usual polar angles of magnetic dipole $\boldsymbol{M}$ or the axis $\boldsymbol{h}$ of the cone envelope, and the $\vartheta_{0}$ is the cone opening angle (see Fig. 1).

The positional angle of polarization $\chi$ is calculated from the equation

$\tan (2 \chi)=\frac{f_{U}}{f_{Q}}$
The case of non-magnetized envelopes follows from Eq. (4) if one takes $\psi=0$. This case was considered in Dolginov \& Silant'ev (1974) and Brown \& McLean (1977) (note that in the latter paper in the basic formula (23), factor 2 is omitted).

\section{Faraday rotation in turbulent envelopes}

Recently Silant'ev (2005) developed the radiative transfer theory for turbulent magnetized atmospheres and found that the averaged Stokes parameters obey the usual radiative transfer equation with new effective (renormalized) kinetic coefficients. Note that the observed Stokes parameters $I, Q, U$, and $V$ coincide with the statistically averaged quantities if many turbulent "cells" exist in the region of the observation. In turbulent atmosphere all quantities $-\alpha, N_{\mathrm{e}}, \boldsymbol{B}, I, Q, U, V$ are stochastic functions consisting of the regular (mean) and fluctuating terms. We denote the mean values by subscript zero, and the fluctuating terms - by prime (example, $\alpha=\alpha_{0}+\alpha^{\prime}$ with $\alpha_{0} \equiv\langle\alpha\rangle$ and $\left.\left\langle\alpha^{\prime}\right\rangle \equiv 0, N_{\mathrm{e}}=N_{0}+N_{\mathrm{e}}^{\prime}\right)$. Further, we shall only deal with the averaged Stokes parameters $I_{0}, Q_{0}$ and $U_{0}$.

First, the existence of fluctuations manifests itself by the decrease in the extinction factor:

$\alpha_{\text {eff }}=\alpha_{0}\left(1-\frac{\left\langle\alpha^{\prime 2}\right\rangle}{\alpha_{0}^{2}} \tau_{1} f_{\alpha}\right)$.

Here $\tau_{1}=\alpha_{0} R_{1}$ is the mean optical depth of the turbulence correlation length $R_{1}$, and $f_{\alpha}$ is the constant value $\left(f_{\alpha} \cong\right.$ 1) connected with the correlation function $A_{\alpha}\left(R / R_{1}\right)$ of $\alpha^{\prime}$ fluctuations:

$\left\langle\alpha^{\prime}(s) \alpha^{\prime}\left(s^{\prime}\right)\right\rangle=\left\langle\alpha^{\prime 2}\right\rangle A_{\alpha}\left(R / R_{1}\right)$,

$f_{\alpha}=\int_{0}^{\infty} \mathrm{d} x A(x)$

where $R=\left|s-s^{\prime}\right|$ is the difference in coordinates $s$ and $s^{\prime}$ lying at the line of sight. Very often one uses the exponential correlation function $A(x)=\exp (-x)$, for which $f_{\alpha}=1$.

The validity of renormalized radiative transfer equations is $\tau_{1} \ll 1$. Note that $\alpha_{\text {eff }} \neq \alpha_{0}$. For the often used "shortcorrelated" model of turbulence $\left(R_{1} \rightarrow 0\right)$, this effect disappears. In practice, this effect is small and does not change the degree of polarization.

If the envelope plasma is turbulent the Faraday effect gives rise to two new effects. First, the fluctuating Faraday rotations produce a net decrease in the linear polarization of every light beam propagating in such a medium. Statistically we observe the sum of the radiation fluxes with very different angles of the polarization plane. Clearly, the mean polarization of radiation will be smaller than in some of the particular realizations. Remember that, in the medium with regular Faraday rotation, the separate light's beam does not change the initial degree of polarization because intensity $I$ and the Stokes parameters $Q$ and $U$ diminish with the same extinction factor $\alpha$, as is clearly seen from Eqs. (1) and (10). The known depolarization effect for regular Faraday rotations arises when we observe radiation from various volume elements. Magnetic field fluctuations depolarize the radiation at every separate path of the light. This effect occurs even when the magnetic field is almost stochastic. 
The second effect is more subtle, as results from superposition of regular Faraday rotation and fluctuations in the extinction factor. The qualitative explanation (see Silant'ev 2005) is as follows. Let the first realization of the stochastic picture have the optical depth $\tau_{+}=\tau_{0}+\tau^{\prime}>\tau_{0}$. In this case the Faraday rotation of the polarization plane is greater than for a mean path $\tau_{0}$. But simultaneously the magnitude of polarized light intensity (remember that $Q, U \propto \exp \left(-\left(\tau_{0}+\tau^{\prime}\right)\right)$ is less than for optical depth $\tau_{0}$. For the second realization with $\tau_{-}=\tau_{0}-\tau^{\prime}<\tau_{0}$, the Faraday rotation is smaller than the one corresponding to $\tau_{0}$, but the magnitude of polarized intensity is greater. As a result, the effective Faraday rotation angle is smaller than the mean value $\psi_{0}$. If the mean magnetic field $\boldsymbol{B}_{0} \equiv\langle\boldsymbol{B}\rangle=0$, this explanation does not work because, for every realization, there exists magnetic fluctuations $\boldsymbol{B}^{\prime}$ and $-\boldsymbol{B}^{\prime}$ with equal probability, and the net Faraday rotation does not arise.

These two effects can be qualitatively derived from the model of two realizations with the parameters $\tau_{0}+\tau^{\prime}, \psi_{0}+\psi^{\prime}$, and $\tau_{0}-\tau^{\prime}, \psi_{0}-\psi^{\prime}$; for simplicity we consider here that magnetic field $\boldsymbol{B}$ is regular and the fluctuations $\tau^{\prime}$ and $\psi^{\prime}$ are only due to fluctuations of electron number density $N_{\mathrm{e}}^{\prime}$. Indeed, the exact solutions of radiative transfer equations (1) without the source terms are:

$$
\begin{aligned}
& I_{ \pm}(s)=I(0) \mathrm{e}^{-\left(\tau_{0} \pm \tau^{\prime}\right)}, \\
& Q_{ \pm}(s)=Q(0) \mathrm{e}^{-\left(\tau_{0} \pm \tau^{\prime}\right)} \cos 2\left(\chi_{0} \pm \chi^{\prime}\right), \\
& U_{ \pm}(s)=Q(0) \mathrm{e}^{-\left(\tau_{0} \pm \tau^{\prime}\right)} \sin 2\left(\chi_{0} \pm \chi^{\prime}\right),
\end{aligned}
$$

where $I(0), Q(0)$, and $U(0)=0$ are the initial Stokes parameters at $s=0$, and $s$ is the path of radiation in the turbulent magnetized atmosphere. Optical depths and positional angles are the path integrals:

$$
\begin{aligned}
\tau_{0} \pm \tau^{\prime}= & \int_{0}^{s} \mathrm{~d} s \alpha_{0}(s) \pm \int_{0}^{s} \mathrm{~d} s \alpha^{\prime}(s), \\
\chi_{0} \pm \chi^{\prime}= & \frac{1}{2} \sigma_{\mathrm{T}} \int_{0}^{s} \mathrm{~d} s N_{0}(s) \delta_{0}(s) \cos \Theta_{0}(s) \\
& \pm \frac{1}{2} \sigma_{\mathrm{T}} \int_{0}^{s} \mathrm{~d} s N_{\mathrm{e}}^{\prime}(s) \delta_{0}(s) \cos \Theta_{0}(s) .
\end{aligned}
$$

Calculation of mean values $I_{0}(s)=\left(I_{+}+I_{-}\right) / 2$ etc. for small $\tau^{\prime}$ and $\chi^{\prime}$ gives rise to the formulae:

$$
\begin{aligned}
I_{0}(s)= & I(0) \mathrm{e}^{-\tau_{0}} \cosh \left(\tau^{\prime}\right) \cong I(0) \mathrm{e}^{-\left(\tau_{0}-\tau^{\prime 2} / 2\right)} \\
\equiv & I(0) \mathrm{e}^{-\tau_{\text {eff }}}<I(0) \mathrm{e}^{-\tau_{0}}, \\
Q_{0}(s) \cong & Q(0) \mathrm{e}^{-\tau_{0}}\left[\left(1+\frac{\tau^{\prime 2}}{2}-2 \chi^{\prime 2}\right) \cos \left(2 \chi_{0}\right)\right. \\
& \left.+2 \tau^{\prime} \chi^{\prime} \sin \left(2 \chi_{0}\right)\right], \\
U_{0}(s) \cong & Q(0) \mathrm{e}^{-\tau_{0}}\left[\left(1+\frac{\tau^{\prime 2}}{2}-2 \chi^{\prime 2}\right) \sin \left(2 \chi_{0}\right)\right. \\
& \left.-2 \tau^{\prime} \chi^{\prime} \cos \left(2 \chi_{0}\right)\right] .
\end{aligned}
$$

Note that these formulae are valid in the general case where the magnetic field is stochastic with the isotropic distribution of fluctuations. It is seen from Eqs. (12) that the effective optical depth for the intensity

$\tau_{\text {eff }}=\tau_{0}-\frac{1}{2} \tau^{\prime 2}$

is smaller than the mean optical depth $\tau_{0}$. The expressions for $Q_{0}(s)$ and $U_{0}(s)$ show that effective extinction of the polarization magnitude is greater than for the intensity:

$P_{0}(s) \equiv \sqrt{Q_{0}^{2}(s)+U_{0}^{2}(s)}=P(0) \mathrm{e}^{-\left(\tau_{\text {eff }}+2 \chi^{\prime 2}\right)}$.

The additional term $2 \chi^{\prime 2}$ describes the depolarization effect of stochastic Faraday rotations by the propagation of the polarized radiation in a turbulent magnetized medium. This is the statistical effect. The exact expressions (10) demonstrate that $P_{ \pm}(s)=$ $P(0) \exp \left(-\left(\tau_{0} \pm \tau^{\prime}\right)\right)$, i.e. extinction of polarization magnitude is the same as for intensity $I$, and the degree of polarization does not change $\left(p_{ \pm}=P_{ \pm} / I_{ \pm} \equiv P(0) / I(0)=p(0)\right)$. Calculation of $U_{0}(s) / Q_{0}(s) \equiv \tan \left(2 \chi_{\text {eff }}\right)$ gives rise to the expression:

$\chi_{\mathrm{eff}}=\chi_{0}-\tau^{\prime} \chi^{\prime}$

which explains the second effect. Note that $\tau^{\prime} \propto \alpha^{\prime}$ and $\chi^{\prime} \propto$ $N_{\mathrm{e}}^{\prime}$. The term with $\boldsymbol{B}^{\prime}$ in $\chi^{\prime}$ disappears because statistically the fluctuations $\boldsymbol{B}^{\prime}$ and $-\boldsymbol{B}^{\prime}$ occur with equal probability.

Assuming the Gaussian ensemble of realizations all fluctuating values, Silant'ev (2005) derived the new renormalized transfer equations for average Stokes parameters $I_{0}, Q_{0}$, and $U_{0}$ :

$(\boldsymbol{n} \nabla) I_{0}(\boldsymbol{r}, \boldsymbol{n})=-\alpha_{\mathrm{eff}} I_{0}(\boldsymbol{r}, \boldsymbol{n})+\left\langle N_{\mathrm{e}} \sigma_{\mathrm{T}} B_{i}(\boldsymbol{r}, \boldsymbol{n})\right\rangle$,

$(\boldsymbol{n} \nabla) Q_{0}(\boldsymbol{r}, \boldsymbol{n})=-\alpha_{\mathrm{pol}} Q_{0}(\boldsymbol{r}, \boldsymbol{n})$

$-N_{0}(\boldsymbol{r}) \sigma_{\mathrm{T}} \delta_{\mathrm{eff}} \cos \Theta_{0} U_{0}(\boldsymbol{r}, \boldsymbol{n})+\left\langle N_{\mathrm{e}} \sigma_{\mathrm{T}} B_{Q}(\boldsymbol{r}, \boldsymbol{n})\right\rangle$,

$(\boldsymbol{n} \nabla) U_{0}(\boldsymbol{r}, \boldsymbol{n})=-\alpha_{\mathrm{pol}} U_{0}(\boldsymbol{r}, \boldsymbol{n})$

$+N_{0}(\boldsymbol{r}) \sigma_{\mathrm{T}} \delta_{\mathrm{eff}} \cos \Theta_{0} Q_{0}(\boldsymbol{r}, \boldsymbol{n})+\left\langle N_{\mathrm{e}} \sigma_{\mathrm{T}} B_{U}(\boldsymbol{r}, \boldsymbol{n})\right\rangle$.

Here $N_{0}(\boldsymbol{r}) \equiv\left\langle N_{\mathrm{e}}(\boldsymbol{r})\right\rangle$ is the average value of the electron number density. The average source functions are presented in Silant'ev (2005). The effective coefficients $\alpha_{\mathrm{pol}}$ and $\delta_{\text {eff }}$ have the following form:

$$
\begin{aligned}
\alpha_{\mathrm{pol}}= & \alpha_{\mathrm{eff}}+N_{0} \sigma_{\mathrm{T}} \tau_{1}^{(\mathrm{T})} \\
& \times\left[\left\langle\delta^{\prime 2}\right\rangle f_{B}+\delta_{0}^{2} \cos ^{2} \Theta_{0} \frac{\left\langle N^{\prime 2}\right\rangle}{N_{0}^{2}} f_{N}\right], \\
\delta_{\mathrm{eff}}= & \delta_{0}\left[1-2 \tau_{1} \frac{\left\langle\alpha^{\prime} N_{\mathrm{e}}^{\prime}\right\rangle}{\alpha_{0} N_{0}} f_{\alpha N}\right] .
\end{aligned}
$$

Here $\alpha_{\text {eff }}$ is given in Eq. (8), $\tau_{1}^{(\mathrm{T})}=N_{0} \sigma_{\mathrm{T}} R_{1}$ is the mean Thomson optical depth of the turbulent correlation length $R_{1}$, and $\left\langle\delta^{\prime 2}\right\rangle=\left(0.8 \lambda^{2}(\mu \mathrm{m})\right)^{2}\left\langle B^{\prime 2}\right\rangle / 3$ is the rms-value of the fluctuations of the parameter (2). The factors $f_{B}, f_{N}$, and $f_{\alpha N}$ are related to the corresponding correlation functions in Eq. (9). Remember that $\tau_{1}$ is the total optical depth of the correlation length $R_{1}$ including the true absorption. For real envelopes all the effective coefficients depend on the position in an envelope. 
The second term in Eq. (17) describes the first effect - the decrease in the polarization degree due to fluctuations of a magnetic field and the electron number density. The term with $\tau_{1}$ in Eq. (18) characterizes the second effect - decrease in the effective Faraday rotation angle compared with its mean value (remember that $\psi \propto \delta$ ). The terms with $\tau_{1}$ in Eq. (8) and $\tau_{1}^{(\mathrm{T})}$ in Eq. (18) are small, so we neglect them when calculating the polarization from envelopes. The second term in Eq. (17), in contrast, can acquire high values because the parameters $\delta_{0}$ and $\delta^{\prime}$ can be very large (see Eq. (2)). Considering the single scattering of the radiation in optically thin envelopes, we can neglect the small terms in Eqs. (8), (17), and (18), only the second term in Eq. (17) will be taken into account. As a result, we obtain the following formulae for $F_{Q}(\boldsymbol{n})$ and $F_{U}(\boldsymbol{n})$ :

$$
\begin{aligned}
F_{Q}(\boldsymbol{n}) & =-\frac{L}{4 \pi R^{2}} \\
\times & \frac{3 \sigma_{\mathrm{T}}}{16 \pi} \int \mathrm{d} V \frac{N_{0}(\boldsymbol{r})}{r^{2}} \sqrt{1-\frac{R_{\mathrm{s}}^{2}}{r^{2}}} \sin ^{2} \vartheta \cos 2(\varphi+\psi) \mathrm{e}^{-C}, \\
F_{U}(\boldsymbol{n}) & =-\frac{L}{4 \pi R^{2}} \\
\times & \frac{3 \sigma_{\mathrm{T}}}{16 \pi} \int \mathrm{d} V \frac{N_{0}(\boldsymbol{r})}{r^{2}} \sqrt{1-\frac{R_{\mathrm{s}}^{2}}{r^{2}}} \sin ^{2} \vartheta \sin 2(\varphi+\psi) \mathrm{e}^{-C},
\end{aligned}
$$

where $\psi$ is the path integral (5). The analogous expression for $C$ is:

$$
\begin{aligned}
C(\boldsymbol{n}, \boldsymbol{r})= & \sigma_{\mathrm{T}}\left(0.8 \lambda^{2}(\mu \mathrm{m})\right)^{2} \int \mathrm{d} l N_{0}\left(\boldsymbol{r}^{\prime}\right) \tau_{1}^{(\mathrm{T})} \\
& \times\left[\frac{\left\langle B^{\prime 2}\right\rangle}{3} f_{B}+\left(\boldsymbol{B}_{0} \cdot \boldsymbol{n}\right)^{2} \frac{\left\langle N_{\mathrm{e}}^{\prime 2}\right\rangle}{N_{0}^{2}} f_{N}\right] .
\end{aligned}
$$

Here $\boldsymbol{B}_{0}(\boldsymbol{r})$ is the regular component of the magnetic field. Note that the parameter $\left\langle\delta^{2}\right\rangle$ does not depend on the angles in isotropic turbulence where $\left\langle\left(\boldsymbol{B}^{\prime} \cdot \boldsymbol{n}\right)^{2}\right\rangle=\left\langle B^{\prime 2}\right\rangle / 3$. The Faraday rotation angle $\psi$ in Eqs. (19) only depends on the regular component of the magnetic field.

\section{Spherical envelope with a dipole magnetic field}

A spherical envelope with a regular dipole magnetic field $\boldsymbol{B}_{0}(\boldsymbol{r})$

$\boldsymbol{B}_{0}(\boldsymbol{r})=\frac{3(\boldsymbol{M} \cdot \boldsymbol{r}) \boldsymbol{r}-\boldsymbol{M} \boldsymbol{r}^{2}}{r^{5}}$

was consider in detail in Gnedin et al. (2005). Thus, our aim here is to investigate how the polarization spectrum $p(\lambda)$ and the spectrum of the positional angle $\chi(\lambda)$ change under the influence of magnetic field fluctuations $\boldsymbol{B}^{\prime}$ and the electron number density fluctuations $N_{\mathrm{e}}^{\prime}$. It is convenient to choose the direction of magnetic moment $\boldsymbol{M}$ lying in the plane $(X Z)$, where the $Z$-axis is directed along the line of sight to the observer $\boldsymbol{n}$.

The Faraday rotation angle (5) has the following analytical form (see Gnedin et al. 2005):

$$
\begin{aligned}
\psi(\boldsymbol{n}, \boldsymbol{r})= & \frac{\delta_{\mathrm{s}} \tau_{\mathrm{env}}}{30 \rho^{4}}\left\{\frac{\cos \vartheta_{m}}{(1+\cos \vartheta)^{2}}[-4+7 \cos \vartheta\right. \\
& \left.\left.+18 \cos ^{2} \vartheta+9 \cos ^{3} \vartheta\right]+9 \sin \vartheta_{m} \cos \varphi \sin \vartheta\right\} .
\end{aligned}
$$

Here $\delta_{\mathrm{s}}=0.8 \lambda^{2}(\mu \mathrm{m}) M / R_{\mathrm{s}}^{3}$ is the parameter (2) at the magnetic equator of the star surface, $R_{\mathrm{s}}$ is the radius of a star, $\tau_{\text {env }}=N_{\mathrm{s}} \sigma_{\mathrm{T}} R_{\mathrm{S}}$ is the radial optical depth of an envelope, $\rho=r / R_{\mathrm{s}}$ is the dimensionless distance of the volume element in Eqs. (19). For the electron number density we have chosen $N_{0}(r)=N_{\mathrm{s}} / \rho^{2}$. The angles $\vartheta$ and $\vartheta_{m}$ are the usual polar angles between the direction to an observer $\boldsymbol{n}$ and radiusvector $\boldsymbol{r}$ and $\boldsymbol{M}$, respectively, while $\varphi$ is azimuthal angle of $\boldsymbol{r}$ (see Fig. 1).

To investigate the influence of turbulent fluctuations $\boldsymbol{B}^{\prime}$ and $N_{\mathrm{e}}^{\prime}$ on the spectra $p(\lambda)$ and $\chi(\lambda)$, we assume that the level of these fluctuations is constant compared with its mean values. Besides, we assume that the optical depth of turbulent cells $\tau_{1}^{(\mathrm{T})}$ is also constant in the envelope. These are rather natural assumptions and they simplify formulae for $C(\boldsymbol{n}, \boldsymbol{r})$. As a result, we obtain:

$C(\boldsymbol{n}, \boldsymbol{r})=C_{B}(\boldsymbol{n}, \boldsymbol{r})+C_{N}(\boldsymbol{n}, \boldsymbol{r})$,

where $C_{B}$ and $C_{N}$ corresponds to the first and second terms in Eq. (20). The expression $C_{B}$ acquires the form:

$$
\begin{aligned}
C_{B}(\boldsymbol{n}, \boldsymbol{r})= & \gamma_{B} f_{B} \frac{\tau_{1}^{(\mathrm{T})}}{\tau_{\mathrm{env}}} \cdot \frac{\left(\delta_{\mathrm{s}} \tau_{\mathrm{env}}\right)^{2}}{(\rho \sin \vartheta)^{7}} \int_{0}^{\vartheta} \mathrm{d} \vartheta^{\prime} \sin ^{6} \vartheta^{\prime} \\
C_{N}(\boldsymbol{n}, \boldsymbol{r})= & \gamma_{N} f_{N} \frac{\tau_{1}^{(\mathrm{T})}}{\tau_{\mathrm{env}}} \cdot \frac{\left(\delta_{\mathrm{s}} \tau_{\mathrm{env}}\right)^{2}}{(\rho \sin \vartheta)^{7}} \\
& \times \int_{0}^{\vartheta} \mathrm{d} \vartheta^{\prime} \sin ^{6} \vartheta^{\prime}\left[\cos \vartheta_{m}\left(3 \cos ^{2} \vartheta^{\prime}-1\right)\right. \\
& \left.+3 \sin \vartheta_{m} \cos \varphi \sin \vartheta^{\prime} \cos \vartheta^{\prime}\right]^{2}
\end{aligned}
$$

Here we use the notations:

$\gamma_{B}=\frac{\left\langle B_{\mathrm{s}}^{\prime 2}\right\rangle}{B_{\mathrm{s}}^{2}}, \quad \gamma_{N}=\frac{\left\langle N_{\mathrm{e}}^{2}\right\rangle}{N_{\mathrm{s}}^{2}}$,

where $B_{\mathrm{s}}=M / R_{\mathrm{s}}^{3}$ is the characteristic mean magnetic field at the star surface, $\left\langle B_{\mathrm{s}}^{\prime 2}\right\rangle$ is the level of magnetic fluctuations at star's surface $\left(\left\langle B^{\prime 2}(\rho)\right\rangle=\left\langle B_{\mathrm{s}}^{\prime 2}\right\rangle / \rho^{6}\right),\left\langle N_{\mathrm{e}}^{\prime 2}(\rho)\right\rangle=\left\langle N_{\mathrm{e}}^{\prime 2}\right\rangle / \rho^{2}$, and $N_{\mathrm{s}}$ is the mean electron number density at the star surface. The values $\left\langle B_{\mathrm{s}}^{\prime 2}\right\rangle$ and $\left\langle N_{\mathrm{e}}^{\prime 2}\right\rangle$ are the rms values of fluctuations at the star surface, i.e. the parameters $\gamma_{B}$ and $\gamma_{N}$ are independent of $\rho$. The integrals in Eqs. (24) and (25) are standard and can be taken, for example, from Gradshteyn \& Ryzhik (1994).

In Fig. 2 we present the results of the numerical calculations of $p(\lambda) / \tau_{\text {env }}$ and $\chi(\lambda)$ for the case of purely magnetic fluctuations $\left(C_{N}=0\right)$ for various values of parameter $b=$ $\gamma f_{B}\left(\tau_{1}^{(\mathrm{T})} / \tau_{\text {env }}\right)$. Remember that parameter $\sqrt{\delta_{\mathrm{s}} \tau_{\text {env }}} \propto \lambda$; i.e. our figures are the spectra of the polarization degree and the positional angle. In Fig. 3 the same is presented for the case of electron number density fluctuations $\left(C_{B}=0\right)$. The positive values of positional angle $\chi$ correspond to the left-hand rotation from the plane (Mn) if we see along direction $\boldsymbol{n}$ to an observer. For $\vartheta_{m}=90^{\circ}$ the plane of electric field oscillations coincides with (Mn)-plane $(\chi=0)$.

First of all we see that the magnetic fluctuations change the spectra $p(\lambda)$ and $\chi(\lambda)$ very strongly when compared with the case of the electron number density fluctuations. What is the reason for this difference? The difference between $C_{B}(\boldsymbol{n}, \boldsymbol{r})$ 

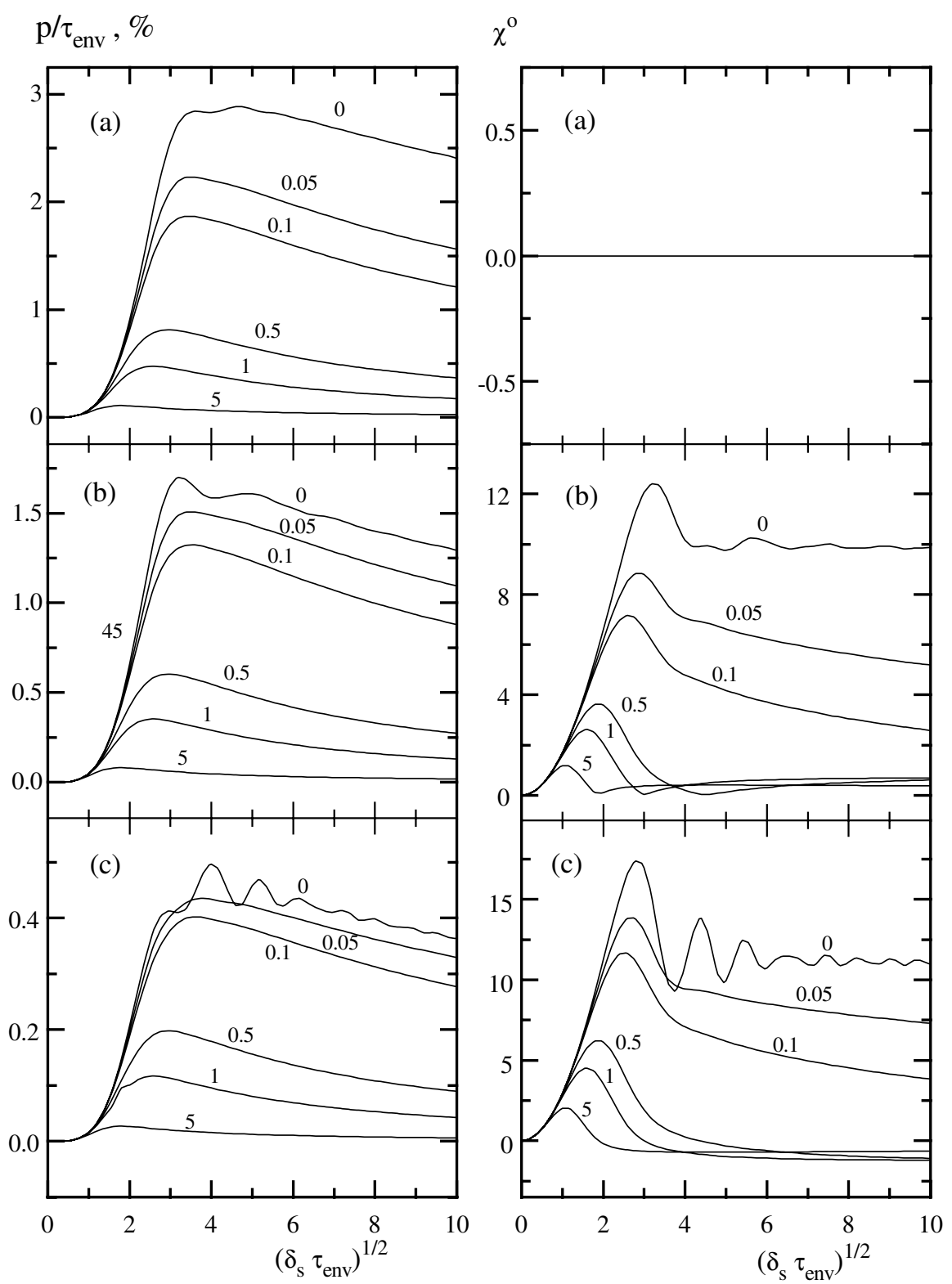

Fig. 2. The degree of polarization and the positional angle of radiation scattered in a spherical envelope with a magnetic dipole field. The cases a), b), and c) correspond to the inclination angles of the magnetic dipole $\boldsymbol{M}: \vartheta_{m}=90^{\circ}, 60^{\circ}$, and $30^{\circ}$. The numbers near curves denote the values of the parameter $b=\left\langle B_{\mathrm{s}}^{\prime 2}\right\rangle / B_{\mathrm{s}}^{2} f_{B}\left(\tau_{1}^{(\mathrm{T})} / \tau_{\text {env }}\right)$.

and $C_{N}(\boldsymbol{n}, \boldsymbol{r})$ consists of the additional term $\cos ^{2} \Theta_{0}$ in expression (25) (the term in square brackets) for $C_{N}$ compared with Eq. (24) for $C_{B}$. Remember that $\Theta_{0}$ is the angle between regular magnetic field $\boldsymbol{B}_{0}$ and the line of sight $\boldsymbol{n}$. The volumes of an envelope with small $\cos \Theta_{0}$ contribute the most to the integral polarization from the envelope, the factor $C_{N}$ is smaller than the $C_{B}$-coefficient. This explains the relatively small contribution of the electron number density fluctuations compared to the contribution from the magnetic field fluctuations. For this reason one can only take the action of magnetic fluctuations into account.

In contrast to the scalar electron number density, the vector magnetic field can have mean value a zero. In this case the $\gamma_{B}$ - parameter in Eq. (24) has to be taken equal to unity, and the parameter $\delta_{\mathrm{s}}^{2}$ is equal to the value $\delta_{\mathrm{s}}^{2}=\left(0.8 \lambda^{2}(\mu \mathrm{m})\right)^{2}\left\langle B_{\mathrm{s}}^{\prime 2}\right\rangle$. Here $\left\langle B_{\mathrm{s}}^{\prime 2}\right\rangle$ is the rms-value of magnetic fluctuations at the surface of a star. If the $\left\langle B_{\mathrm{s}}^{\prime 2}\right\rangle \gg B_{\mathrm{s}}^{2}$, then the $b$-parameter can be very large. The behavior of the positional angle $\chi$ in both cases is qualitatively the same. Note that $\chi \rightarrow 0$ for $\sqrt{\delta_{\mathrm{s}} \tau_{\mathrm{env}}} \rightarrow \infty$. For an envelope without any fluctuations $\chi \rightarrow 11.25^{\circ}$ (see Sect. 6).

The spherical envelope without the regular magnetic field can give the integral polarization if the distribution of magnetic fluctuations $\left\langle B^{\prime 2}(\boldsymbol{r})\right\rangle$ is not axially symmetric along the line of sight $\boldsymbol{n}$.

\section{Polarization from magnetized turbulent cone-like jets}

Cone-like jets possess integral polarization (see, Dolginov \& Silant'ev 1974 and Dolginov et al. 1995) even without any 

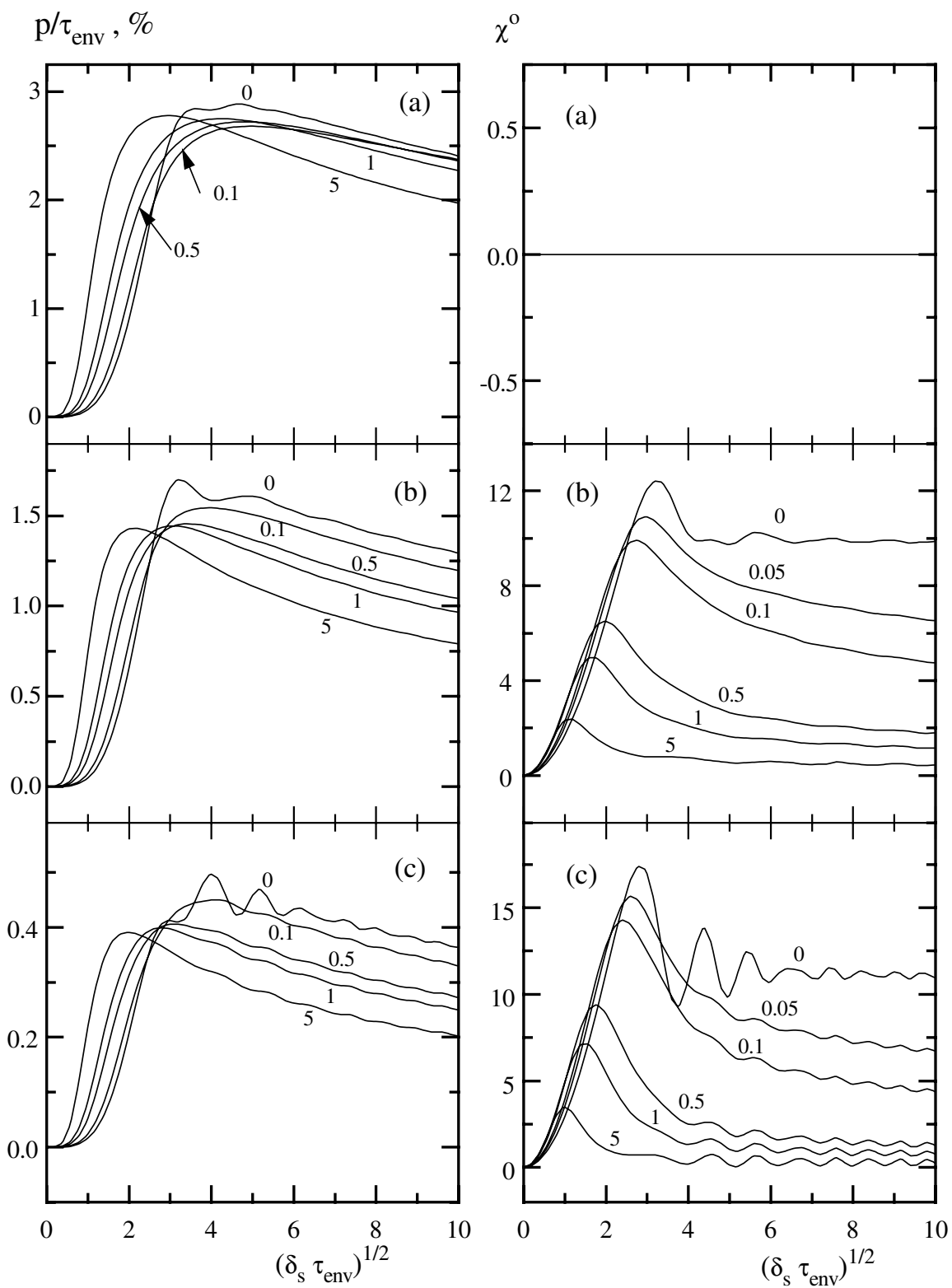

Fig. 3. Same as in Fig. 2 for the turbulent electron number density fluctuations. Parameter $b=\left\langle N_{\mathrm{e}}^{\prime 2}\right\rangle / N_{\mathrm{s}}^{2} f_{N}\left(\tau_{1}^{(\mathrm{T})} / \tau_{\text {env }}\right)$.

magnetic field, due to the absence of the axial symmetry of the scattering picture:

$p=\frac{\left|F_{Q}\right|}{L / 4 \pi R^{2}}=\frac{3}{16} \cdot \frac{\pi}{4} \tau_{\text {env }} \cos \vartheta_{0} \sin ^{2} \vartheta_{0} \sin ^{2} \vartheta_{h}$

where $\vartheta_{h}$ is the polar angle of the cone-axis $\boldsymbol{h}$, and $\vartheta_{0}$ the cone opening angle (see Fig. 1). The factor $\pi / 4$ arises when we take into account that the star is not a point-like source of radiation (for the case $N_{\mathrm{e}} \propto r^{-2}$ and $I(\mu)=$ const.). Thus, Eq. (27) gives the lower limit for the polarization degree. The electric field oscillations of scattered radiation are perpendicular to the plane ( $\boldsymbol{n h}$ ) that corresponds to $\chi=0$ in Fig. 4 . The value $\tau_{\text {env }}$ is the radial Thomson optical depth of the cone-like envelope. For the model with $N_{0}(r)=N_{\mathrm{s}}\left(R_{\mathrm{s}}^{2} / r^{2}\right)$, it is equal to $N_{\mathrm{s}} \sigma_{\mathrm{T}} R_{\mathrm{s}}$.

The integration in formulae (19) can be easily performed using the coordinate system $(\boldsymbol{h})$ with $Z$-axis along the symmetry axis of a cone and with $X$-axis lying in the plane (nh). The radius-vector $\boldsymbol{r}$ of the volume element $d V$ in this system is characterized by $(r, \vartheta, \varphi)$, and in the system $(\boldsymbol{n})$ with $Z$-axis along $\boldsymbol{n}$ by $(r, \theta, \phi)$. According to formulae of spherical geometry, one has the relations:

$\cos \theta=\cos \vartheta \cos \vartheta_{h}-\sin \vartheta \sin \vartheta_{h} \cos \varphi$

$\cos \vartheta=\cos \theta \cos \vartheta_{h}+\sin \theta \sin \vartheta_{h} \cos \phi$

$\sin \theta \sin \phi=\sin \vartheta \sin \varphi$

The Faraday rotation angle $\psi(\boldsymbol{n}, \boldsymbol{r})$ (see Eq. (5)) can be presented analytically for the following forms of the electron number density and the mean magnetic field:

$N_{0}(r)=\frac{N_{\mathrm{s}}}{\rho^{n}}, \quad \boldsymbol{B}_{0}(\boldsymbol{r})=\frac{B_{\mathrm{s}}}{\rho^{m}} \boldsymbol{e}_{r}$, 

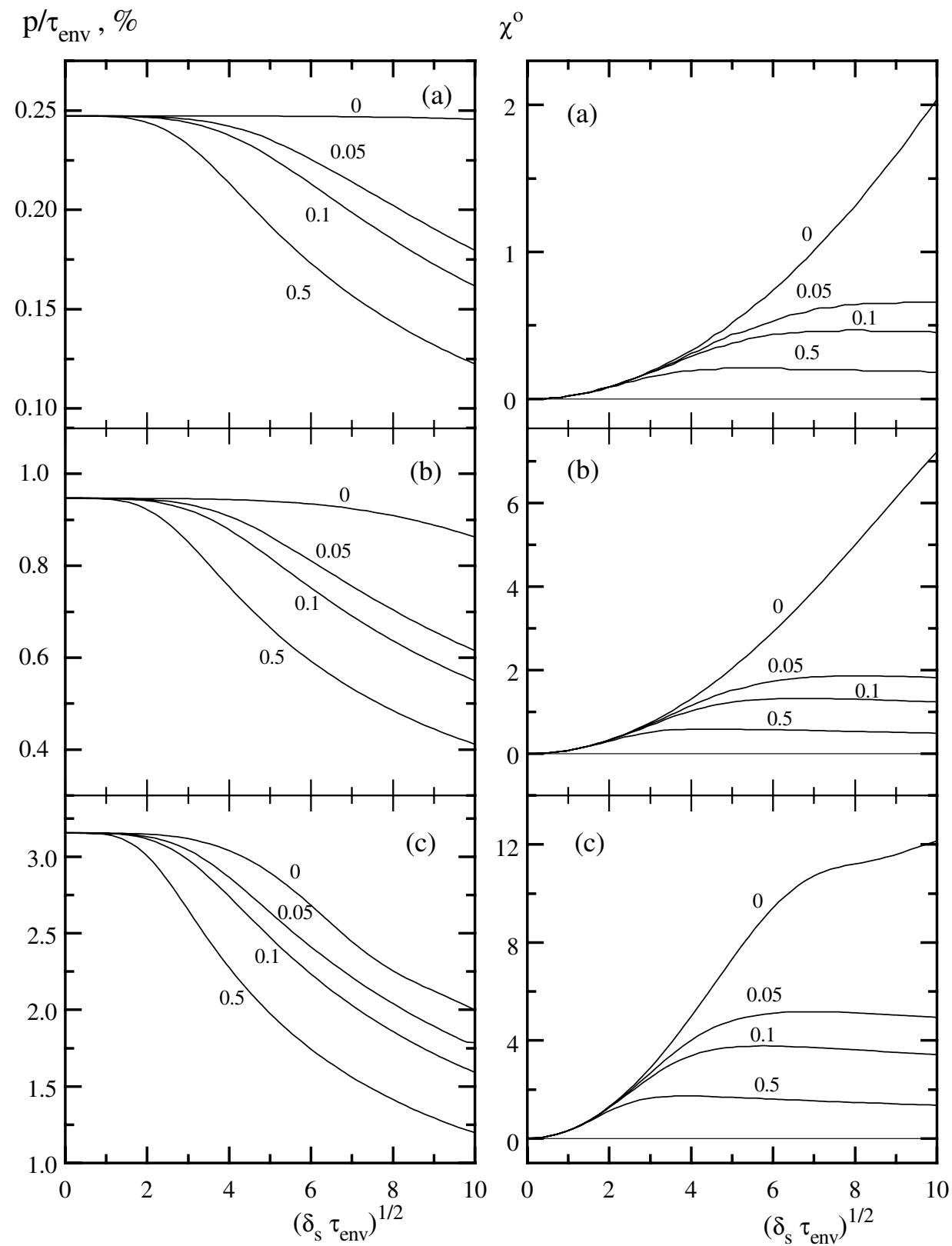

Fig. 4. The degree of polarization and the positional angle of the radiation scattered in a cone-like envelope with $\vartheta_{h}=90^{\circ}$. The cases a), b), and c) correspond to $\vartheta_{0}=7.5^{\circ}, 15^{\circ}$ and $30^{\circ}$. The numbers near curves denote the values of parameter $b=\left\langle B_{\mathrm{s}}^{\prime 2}\right\rangle / B_{\mathrm{s}}^{2} f_{B}\left(\tau_{1}^{(\mathrm{T})} / \tau_{\mathrm{env}}\right)$.

where $\boldsymbol{e}_{r}=\boldsymbol{r} / r$ is the radial unit vector, and $\rho=r / R_{\mathrm{S}}$ is the dimensionless distance from the center of a star. We obtain the following expression:

$\psi(\boldsymbol{n}, \boldsymbol{r})=\frac{\delta_{\mathrm{s}} \tau_{\mathrm{env}}(n-1)}{2(n+m-1) \rho^{n+m-1}}\left[1-\left(\frac{\sin \theta_{b}}{\sin \theta}\right)^{n+m-1}\right]$

Here $\delta_{\mathrm{s}}=0.8 \lambda^{2}(\mu \mathrm{m}) B_{\mathrm{s}}$ is parameter (2) at the surface of a star, and the angle $\theta_{b}$ corresponds to the point where the line of sight transverse the boundary of the envelope. The expression for $\tau_{\text {env }}$ acquires the form:

$\tau_{\text {env }}=\frac{N_{\mathrm{s}} \sigma_{\mathrm{T}} R_{\mathrm{s}}}{n-1}$.

This expression is valid for $n \geq 2$. Our numerical calculation will be made for $n=2$ and $m=2$. General expressions (30) and (31) are then used to obtain the asymptotic behavior of $p(\lambda)$ for large $\lambda$.

Using the formulae (28) we find the expression:

$\cos \theta_{b}=\frac{g+\sqrt{g^{2}-a c}}{a}$

where the values $a, c$, and $g$ are:

$a=\sin ^{2} \theta-\sin ^{2} \vartheta \sin ^{2} \vartheta_{h} \sin ^{2} \varphi$,

$c=\sin ^{2} \theta\left(\cos ^{2} \vartheta_{h} \cos ^{2} \vartheta_{0}-\sin ^{2} \vartheta_{h} \sin ^{2} \vartheta_{0}\right)$

$+\sin ^{2} \vartheta_{h} \sin ^{2} \vartheta \sin ^{2} \varphi$

$g=\sin ^{2} \theta \cos \vartheta_{h} \cos \vartheta_{0}$ 
If the cone-like jet is perpendicular to the line of sight $\left(\vartheta_{h}=\right.$ $90^{\circ}$ ), the polarization of radiation acquires the maximum. For this case one has the relation $\sin \theta_{b} \cos \vartheta=\sin \theta \cos \vartheta_{0}$, and the $\psi$-expression is simpler:

$\psi(\boldsymbol{n}, \boldsymbol{r})=\frac{\delta_{\mathrm{s}} \tau_{\mathrm{env}}(n-1)}{2(n+m-1) \rho^{n+m-1}}\left[1-\left(\frac{\cos \vartheta_{0}}{\cos \vartheta}\right)^{n+m-1}\right]$.

Note that if the angle $\vartheta_{0} \geq \vartheta_{h}$ and $\vartheta_{h} \leq 90^{\circ}$, then the equality $\theta_{b}=0$ takes place.

We have found in the previous section that the electron number density fluctuations give a rather small decrease of the integral polarization compared to the magnetic field fluctuations. For this reason we only consider the latter here. The general expression for $C_{B}$ has the form:

$C_{B}(\boldsymbol{n}, \boldsymbol{r})=b \frac{(n-1)\left(\delta_{\mathrm{s}} \tau_{\mathrm{env}}\right)^{2}}{3(\rho \sin \theta)^{n+2 m-1}} \int_{\theta_{b}}^{\theta} \mathrm{d} \theta^{\prime}\left(\sin \theta^{\prime}\right)^{n+2 m-2}$,

where $b=\left(\gamma_{B} f_{B}\right)\left(\tau_{1}^{(\mathrm{T})} / \tau_{\text {env }}\right)$ with $\gamma_{B}$ given in Eq. (26). If the regular magnetic field is absent, we assume that $\left\langle B^{\prime 2}\right\rangle=$ $\left\langle B_{\mathrm{s}}^{\prime 2}\right\rangle / \rho^{2 m}$ and $\delta_{\mathrm{s}}^{2}=\left(0.8 \lambda^{2}\right)^{2}\left\langle B_{\mathrm{s}}^{\prime 2}\right\rangle$. In this case $b=f_{B} \tau_{1}^{(\mathrm{T})} / \tau_{\mathrm{env}}$.

The results of calculations for the model (29) with $n=2$ and $m=2$ are presented in Fig. 4. We see that the spectra of polarization (remember that $\sqrt{\delta_{\mathrm{s}} \tau_{\mathrm{env}}} \propto \lambda$ ) strongly change its forms compared with ones without the turbulent fluctuations $(b=0)$. Note that these spectra are practically independent of regular magnetic field for all the parameters $b$ mentioned above. This means that the mechanism of stochastic Faraday depolarization for $\sqrt{\delta_{\mathrm{s}} \tau_{\text {env }}} \geq 1-2$ is more effective than the usual depolarization due to various rotation angles from different volumes of the envelope. The positive positional angles $\chi$ correspond to right-hand rotation from the axis $Y$ (see Fig. 1) if we see along the direction $\boldsymbol{n}$ to an observer. The cone jet without the regular magnetic field has $\chi=0$ because the magnetic fluctuations are assumed to be isotropic. Thus, non-zero values of $\chi$ are due to the existence of a regular magnetic field. The spectra $\chi(\lambda)$ are more sensitive to the existence of a regular magnetic field than the $p(\lambda)$-spectra. Note that $\chi(\lambda) \rightarrow 0$ for the turbulent envelope at $\sqrt{\delta_{\mathrm{s}} \tau_{\mathrm{env}}} \rightarrow \infty$, and it tends to $15^{\circ}$ for the envelope with a pure regular magnetic field (see next section).

If we have two equal cone-like jets with the opposite axis, then for $\vartheta_{h}=90^{\circ}$ the total polarization will be twice larger with the same positional angle. For $\vartheta_{h} \neq 90^{\circ}$ the situation is more complex. For many cases with $\vartheta_{h} \geq \vartheta_{0}$ one can roughly take the degree of polarization also is twice larger than for one jet and the net positional angle to be near zero because the oppositely inclined jets have opposite signs of $\chi$.

\subsection{Optically thick narrow jet-like envelopes}

The maximum polarization $p_{\max }\left(\vartheta_{h}, \vartheta_{0}, \tau_{\text {env }}\right)$ from the conelike envelope corresponds to the absence of any magnetic field (see Eq. (27)). The upper limit for $p_{\max }$ takes place for a point-like star at $\vartheta_{h}=90^{\circ}$ and $\vartheta_{0} \cong 55^{\circ}\left(\cos \vartheta_{0}=1 / \sqrt{3}\right)$ : $p_{\text {max }} \cong 7.2 \tau_{\text {env }} \%$. Remember that $\tau_{\text {env }}$ in Eq. (27) is the radial Thomson optical depth of the cone-like envelope, and it was assumed $\tau_{\text {env }} \ll 1$. Thus, for $\tau_{\text {env }}=0.2$ the upper limit polarization is equal to $\approx 1.4 \%$. For angles $\vartheta_{0}=30^{\circ}, 15^{\circ}$, and $7.5^{\circ}$, the polarization degree is smaller: $0.8 \%, 0.24 \%$, and $0.06 \%$, respectively. These are rather low values of polarization (but quite observable by modern polarimeters).

In reality the cone-like envelopes with small $\vartheta_{0}\left(\vartheta_{0} \leq\right.$ $30^{\circ}$ ) can produce greater polarization. For these envelopes the second scattering of radiation can be negligible even at $\tau_{\text {env }} \approx 0.5-2$ (depending on the cone opening angle $\vartheta_{0}$ ). Simultaneously, such envelopes have small optical depth in the direction perpendicular to the axis $\boldsymbol{h}$, so for our case $N_{\mathrm{e}} \propto 1 / r^{2}$ and $\vartheta_{h}=90^{\circ}$, the maximum value for this optical depth is equal to $\tau_{\perp \max }(\rho)=\tau_{\text {env }} \vartheta_{0} / \rho\left(\rho=r / R_{\mathrm{s}}\right.$ is dimensionless distance from the center of a star). The value $\tau_{\perp \max }(\rho)$ corresponds to the perpendicular distance lying in the plane $(\boldsymbol{n h})$ from the axis $\boldsymbol{h}$ of an envelope. Note that the radial optical depth $\tau_{\text {env }}(\rho)=\tau_{\text {env }}(1-1 / \rho)$. It acquires the value $\tau_{\text {env }} / 2$ at $\rho=2$, which is the characteristic distance where most single scatterings occur. For $\vartheta=30^{\circ}, 15^{\circ}$, and $7.5^{\circ}$ and $\tau_{\text {env }}=1$, the characteristic values $\tau_{\perp \max }(2)$ are equal to $0.26,0.13$, and 0.065 , respectively.

To estimate the contribution of multiple scattered radiation (for narrow envelopes this means second scattered photons), we have to calculate the values $F_{I}(\boldsymbol{n})$ and $F_{Q, U}(\boldsymbol{n})$ (see Eqs. (4)) with the additional factors $\exp \left(-\tau_{\text {env }}(\rho)\right)$ and $\exp \left(-\tau_{\perp}(\rho, \vartheta, \varphi)\right)$. The first factor describes the extinction of star's radiation intensity by virtue of its propagation into a cone-like envelope. The second factor describes the extinction of single- scattered radiation by its escaping from the envelope. Below we consider the point-like star with the envelope perpendicular to the line of sight $\left(\vartheta_{h}=90^{\circ}\right)$. The explicit formula for $\tau_{\perp}$ is:

$$
\begin{aligned}
\tau_{\perp}(\rho, \vartheta, \varphi)= & \frac{\tau_{\mathrm{env}}}{\rho \cos \vartheta} \cdot \frac{1}{\sqrt{1+\tan ^{2} \vartheta \sin ^{2} \varphi}} \\
& \times\left[\arctan \frac{\sqrt{\tan ^{2} \vartheta 0 \tan ^{2} \vartheta \sin ^{2} \varphi}}{\sqrt{1+\tan ^{2} \vartheta \sin ^{2} \varphi}}\right. \\
& \left.+\arctan \frac{\tan \vartheta \cos \varphi}{\sqrt{1+\tan ^{2} \vartheta \sin ^{2} \varphi}}\right] .
\end{aligned}
$$

Due to the narrowness of the cone-like envelopes, the probability of the second scattering in directions along the axis is rather small, and is proportional to the small solid angle $\pi \vartheta_{0}^{2}$. The probability is higher of the second scattering into the vast solid angles perpendicular to the cone axis $\boldsymbol{h}$. Physically $\tau_{\perp}(\rho, \vartheta, \varphi)$ is determined by the second scattering events occuring before escaping the envelope. The comparison of the parameters $F_{I}(\boldsymbol{n})$ and $F_{Q}(\boldsymbol{n})$ calculating without the factor $\exp \left(-\tau_{\perp}\right)$ with those taking into account this factor, demonstrate that for $\tau_{\text {env }}=2$ the relative difference is equal to 15,31 , and $66 \%$ for the angles $\vartheta_{0}=7.5,15$, and $30^{\circ}$, respectively. At $\tau_{\text {env }}=1$ these differences are $6.5,13.3$, and $28 \%$. For $\tau_{\text {env }}=0.5$ they are smaller $-3,6$, and $13 \%$, respectively. Thus, we can neglect the secondly scattered photons for $\tau_{\text {env }} \leq 0.5$ for envelopes with $\vartheta_{0} \simeq 30^{\circ}$. For the envelopes with $\vartheta_{0}=15$ and $7.5^{\circ}$ 
the corresponding $\tau_{\text {env }}$ are equal to 1 and 2 . This means that we can neglect the factor $\exp \left(-\tau_{\perp}\right)$ for lower values $\tau_{\text {env }}$ than those mentioned above. Note that the relative differences of the parameters $F_{I}$ and $F_{Q}$ are practically the same. Of course, we take the factor $\exp \left(-\tau_{\text {env }}(\rho)\right)$ into account in all the cases. Due to a wider variety of the scattering angles, the secondly scattered photons contribute less to the integral polarization from the envelopes than do the single-scattered ones.

To take the large $\tau_{\text {env }}(\rho)$ into account in narrow cone-like envelopes, we include in the integrands of Eqs. (4) and (19) the additional term $\exp \left(-\tau_{\text {env }}(\rho)\right)$ that describes the extinction of incident light inside the envelope by the radial propagation. For the case of non-magnetized envelopes with an illuminating point-like star, this changes Eq. (27) as follows:

$p=\frac{3}{16}\left(1-\mathrm{e}^{-\tau_{\text {env }}}\right) \cos \vartheta_{0} \sin ^{2} \vartheta_{0} \sin ^{2} \vartheta_{h}$.

This expression is valid for all models of the electron number density $N_{\mathrm{e}}(r)$. For $\tau_{\text {env }} \ll 1$, formula (37) coincides with Eq. (27) for a point-like star (without the factor $\pi / 4$ ). The term $\exp \left(-\tau_{\perp}\right)$ was omitted by derivation of formula (37), which means that we restict ourselves to the conditions mentioned above: $\tau_{\text {env }} \leq 0.5$ for $\vartheta_{0} \leq 30^{\circ}, \tau_{\text {env }} \leq 1$ for $\vartheta_{0} \leq 15^{\circ}$ and $\tau_{\text {env }} \leq 2$ for $\vartheta_{0} \leq 7.5^{\circ}$. Of course, the angle $\vartheta_{h}$ needs to be near the value $90^{\circ}$. Comparison of Eqs. (37) and (27) shows that Eq. (27) can be used up to $\tau_{\text {env }} \approx 0.5$.

The substitution of terms $\exp (-\tau(\rho))$ and $\exp \left(-\tau_{\perp}(\rho, \vartheta, \varphi)\right)$ into Eqs. (19) gives the polarization for magnetized cone-like narrow envelopes with large radial optical depth. We calculated this polarization for a point-like star. This corresponds to the upper limit for polarization. The results of calculations are presented in Fig. 5, where all the parameters are the same as in Fig. 4. Thus, these figures present the lower and upper limits of polarization.

\section{Spectrum of polarization at large wavelengths}

The dependence of values $\psi(\boldsymbol{n}, \boldsymbol{r})$ and $C(\boldsymbol{n}, \boldsymbol{r})$ on the wavelength $\lambda$ is different: $\psi \propto \lambda^{2}$ and $C \propto \lambda^{4}$. It means that at large $\lambda$ (i.e. at large $\delta_{\mathrm{s}} \tau_{\text {env }}$ ), the decrease in polarization due to chaotic Faraday rotations (see expression (17) for $\alpha_{\text {pol }}$ ) at every light path is more effective than the decrease in integral polarization due to different regular Faraday rotations from various volume elements. First we obtain the wavelength dependence of $p(\lambda)$ when the turbulence is absent $(C(\boldsymbol{n}, \boldsymbol{r})=0)$. Clearly, in this case the main contribution to the integral polarization arises from far parts of an envelope where Faraday rotation is not very strong. In these parts the star can be considered as a point-like source of radiation. Our derivation uses the transition to a new dimensionless distance $x=\left(\delta_{\mathrm{s}} \tau_{\text {env }}\right)^{1 /(n+m-1)} / \rho$ (see expression (34) for $\psi(\boldsymbol{n}, \boldsymbol{r})$ ). The corresponding $x$-integrals in Eqs. (4) will be from zero up to a high value $\left(\delta_{\mathrm{s}} \tau_{\text {env }}\right)^{1 /(n+m-1)}$ that we replace by $\infty$. As a result, we obtain the $\lambda$-dependence:

$p(\lambda) \propto \lambda^{-\frac{2(n-1)}{n+m-1}}, \quad\left(\delta_{\mathrm{s}} \tau_{\mathrm{env}}\right)^{1 /(n+m-1)} \gg 1$.

For the electron number density $N_{0}=N_{s} / \rho^{2}(n=2)$ used in our calculations, we have

$p(\lambda) \propto \frac{1}{\sqrt{\lambda}}$ for any envelope with a dipole magnetic field $(m=3)$, and

$p(\lambda) \propto \frac{1}{\lambda^{2 / 3}}$

for a cone-like envelope with the radial magnetic field $\boldsymbol{B}_{0}(\boldsymbol{r})=$ $\boldsymbol{e}_{r} B_{\mathrm{s}} / \rho^{2}(m=2)$. Remember that Eqs. (38)-(40) describe $p(\lambda)$ for those envelopes without turbulence.

For turbulent envelopes, the exponential term $\exp (-C)$ in Eqs. (19) varies more rapidly with the radius than do the terms with $\psi(\boldsymbol{n}, \boldsymbol{r}) \propto\left(\delta_{\mathrm{s}} \tau_{\text {env }}\right)$, because $C(\boldsymbol{n}, \boldsymbol{r}) \propto\left(\delta_{\mathrm{s}} \tau_{\mathrm{env}}\right)^{2}$. In this case we introduce the new distance $x=\left(\delta_{\mathrm{s}} \tau_{\text {env }}\right)^{2 /(n+2 m-1)} / \rho$ and take $\psi=0$ in Eqs. (19). This gives the following $\lambda$-dependence:

$p(\lambda) \propto \lambda^{-\frac{4(n-1)}{n+2 m-1}}, \quad\left(\delta_{\mathrm{s}} \tau_{\mathrm{env}}\right)^{2 /(n+2 m-1)} \gg 1$.

For the electron number density $N_{0}=N_{\mathrm{s}} / \rho^{2}$ used in our calculations we have

$p(\lambda) \propto \frac{1}{\lambda^{4 / 7}}$

for any turbulent envelope with dipole magnetic field $(m=3)$, and

$p(\lambda) \propto \frac{1}{\lambda^{4 / 5}}$

for a cone-like turbulent envelope with the radial magnetic field $\boldsymbol{B}_{0}(\boldsymbol{r})=\boldsymbol{e}_{r} B_{\mathrm{s}} / \rho^{2}(m=2)$.

Taking $\psi=0$ in Eqs. (19), we obtain $F_{U}=0$ and, as a result, the positional angle $\chi=0$; i.e. for large $\lambda$ the effective electric field oscillations are perpendicular to the plane $(\boldsymbol{M n})$ for the spherical envelope with a magnetic dipole field, and are perpendicular to $(\boldsymbol{h n})$ for cone-like jet.

For the case of non-turbulent envelopes with $N_{0}=N_{\mathrm{s}} / \rho^{2}$, we can demonstrate that $\chi \rightarrow \pi /(4(m+1))$ for a large $\lambda$. For this case the Faraday rotation angle $\psi(\boldsymbol{n}, \boldsymbol{r})$ acquires the general form:

$\psi(\boldsymbol{n}, \boldsymbol{r})=\frac{\delta_{\mathrm{s}} \tau_{\mathrm{env}}}{\rho^{m+1}} \cdot K(\vartheta, \varphi)$.

Function $K(\vartheta, \varphi)$ also depends on other parameters of an envelope, for example, $\vartheta_{m}$ or $\vartheta_{h}$. Let us introduce the new variable $x=\left[\delta_{\mathrm{s}} \tau_{\mathrm{env}} K(\vartheta, \varphi)\right]^{1 /(m+1)} / \rho$. The $\rho$-integration is replaced by $x$-integration with the limits $\left(0,\left(\delta_{\mathrm{s}} \tau_{\text {env }} K(\vartheta, \varphi)^{1 /(m+1)}\right)\right.$. If we take $\infty$ instead of the upper limit, then the Stokes parameters $F_{Q}$ and $F_{U}$ in Eqs. (4) will differ only by the different $x$-integrals:

$F_{Q} \propto \int_{0}^{\infty} \mathrm{d} x \cos \left(x^{m+1}\right)=\Gamma\left(1+\frac{1}{m+1}\right) \cos \frac{\pi}{2(m+1)}$,

$F_{U} \propto \int_{0}^{\infty} \mathrm{d} x \sin \left(x^{m+1}\right)=\Gamma\left(1+\frac{1}{m+1}\right) \sin \frac{\pi}{2(m+1)}$.

The values of the integrals were taken from Gradshtein \& Ryzhik (1994), and $\Gamma(x)$ is the gamma-function. According to Eq. (7) we obtain

$\chi_{\lim }=\frac{\pi}{4(m+1)}$.

For a non-turbulent spherical envelope with the dipole magnetic field $(m=3)$, this formula gives $\chi_{\lim }=11.25^{\circ}$, and 

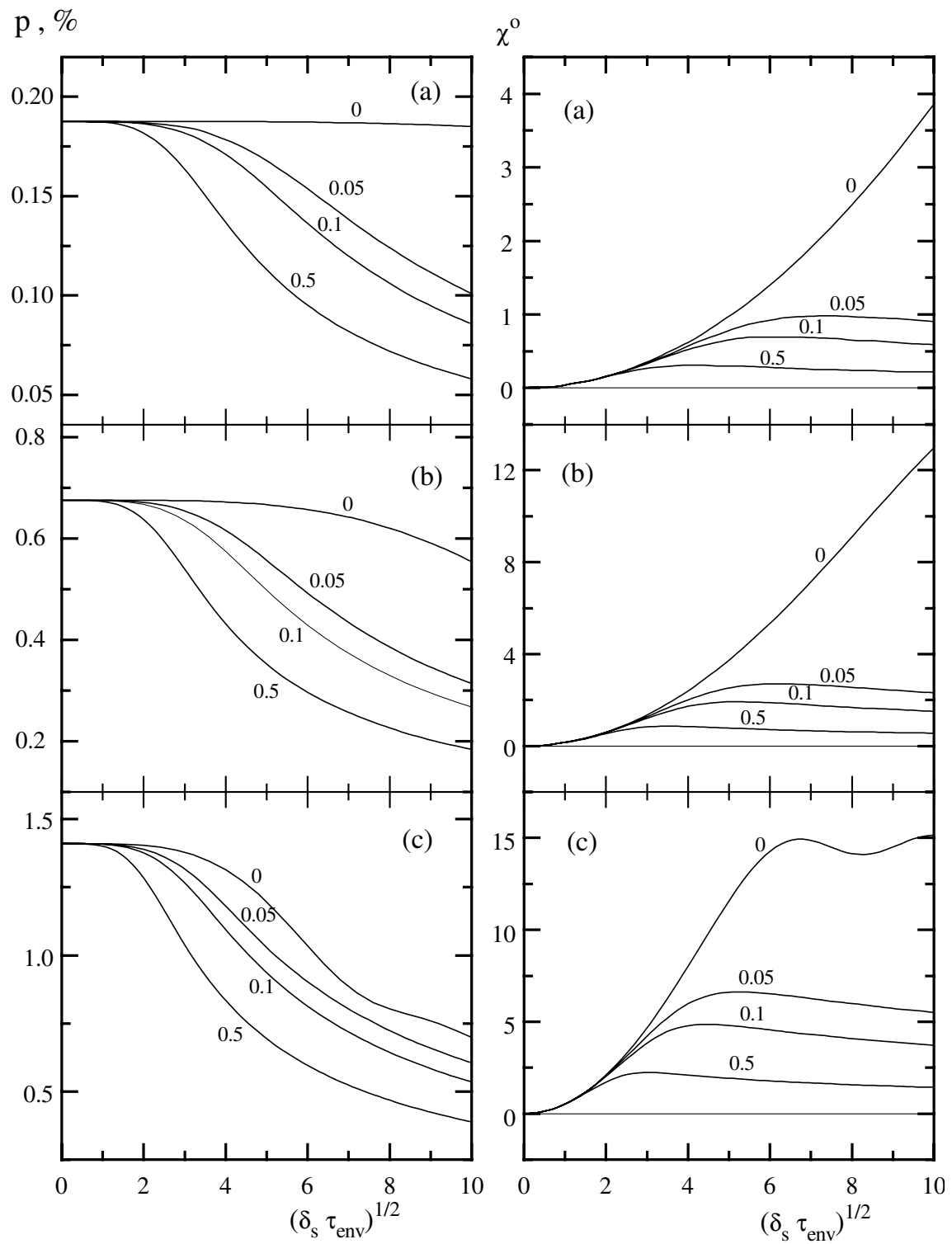

Fig. 5. The degree of polarization and the positional angle of the radiation scattered in a cone-like narrow envelope with $\vartheta_{h}=90^{\circ}$ and $\tau_{\text {env }}=1, \vartheta_{0}=7.5^{\circ}$ (case a)), $\tau_{\text {env }}=1, \vartheta_{0}=15^{\circ}$ (case $\left.\mathbf{b}\right)$ ), and $\tau_{\text {env }}=0.5, \vartheta_{0}=30^{\circ}$ (case $\left.\mathbf{c}\right)$ ). The numbers near curves denote the values of the parameter $b=\left\langle B_{\mathrm{s}}^{2}\right\rangle / B_{\mathrm{s}}^{2} f_{B}\left(\tau_{1}^{(\mathrm{T})} / \tau_{\mathrm{env}}\right)$.

for cone-like jet with radial magnetic field $(m=2)$ one has $\chi_{\lim }=15^{\circ}$. Note that this asymptotic behavior does not depend on the geometry of the envelope, but is affected instead by the radial dependencies of the electron density and the magnetic field.

Thus, by analyzing the observed functions $p(\lambda)$ and $\chi(\lambda)$ for large $\lambda$, we can determine if turbulence exists in an envelope. Then one can reconstruct the $\rho$-dependence of $N_{0}$ and $\boldsymbol{B}_{0}$ and estimate the level of magnetic fluctuation $b$.

For cone-like jets the asymptotic behavior (43) takes place for $\sqrt{\delta_{\mathrm{s}} \tau_{\text {env }}} \geq 10-20$, whereas the asymptotic law (40) for the regular magnetic field occurs for higher values of this parameter $\sqrt{\delta_{\mathrm{s}} \tau_{\text {env }}} \geq 100$. For this reason the difference between these two cases can be established even in the range $\sqrt{\delta_{\mathrm{s}} \tau_{\text {env }}} \approx 5-10$. The narrow cone-like jets with $\tau_{\text {env }} \leq 0.5-2$ (see Sect. 5.1) have the same asymptotical behavior.

\section{Conclusion}

In this paper we investigate the influence of the fluctuations of a magnetic field $\boldsymbol{B}^{\prime}$ and the electron number density $N_{\mathrm{e}}^{\prime}$ on the polarization degree spectra $p(\lambda)$ and the positional angle spectra $\chi(\lambda)$ of the radiation that is single-scattered in a magnetized turbulent circumstellar envelope. The envelopes are assumed to be optically thin and the observed integral polarization of the scattered radiation is calculated. Two models of the envelopes were considered: a spherical envelope with the magnetic dipole field and the cone-like jet with the radial magnetic field. We take into account that the central source ("star") is not a pointlike source of non-polarized radiation and have excluded the contribution of the invisible part of an envelope. The assumed star surface intensity $I(\mu)=$ const. corresponds to a lower limit for possible polarization. In contrast, the model of a point-like source gives the upper limit for polarization. It is shown that 
in narrow cone-like $\left(\vartheta_{0} \leq 30^{\circ}\right)$ envelopes the second scattering of radiation is negligible up to a radial optical thickness of $\tau_{\text {env }} \cong 0.5-2$ (depending of the cone opening angle $\vartheta_{0}$ ). In this case the single - scattered radiation produces maximum polarization $p \simeq 1.4 \%$ at $\tau_{\text {env }}=0.5$ (see Fig. 5).

The magnetic field $\boldsymbol{B}(\boldsymbol{r})=\boldsymbol{B}_{0}(\boldsymbol{r})+\boldsymbol{B}^{\prime}(\boldsymbol{r})$ and the concentration of the plasma electrons $N_{\mathrm{e}}(\boldsymbol{r})=N_{0}(\boldsymbol{r})+N_{\mathrm{e}}^{\prime}(\boldsymbol{r})$ are considered as a sum of the mean values and fluctuating components (denoted by primes). To investigate the problem we have used the recently published theory of radiative transfer in turbulent magnetized atmospheres (Silant'ev 2005). According to this theory the chaotic Faraday rotations due to the fluctuations $\boldsymbol{B}^{\prime}$ and $N_{\mathrm{e}}^{\prime}$ in a magnetized atmosphere give rise to the additional decrease in the degree of linear polarization $p(\lambda)$ of the propagating radiation.

The numerical calculations demonstrate that the influence of the electron concentration fluctuations is less than the contribution of magnetic fluctuations. The additional extinction coefficient for Stokes parameters $Q$ and $U$ is proportional to $\lambda^{4}$, whereas the Faraday rotation angle in a regular magnetic field $\propto \lambda^{2}$. For this reason for large $\lambda$, the extinction of polarized components on every light path is more effective than the integral depolarization due to different Faraday rotations from various volume elements of an envelope. This changes the spectra $p(\lambda)$ and $\chi(\lambda)$ a lot compared with the non-turbulent magnetized envelopes. For large $\lambda$ the polarization $p(\lambda)$ tends to zero much more rapidly than in the envelopes with only the regular magnetic field (see Figs. 2-5). We present asymptotic formulae for $p(\lambda)$ at large $\lambda$ for various models of $\boldsymbol{B}(\boldsymbol{r})$ and $N_{\mathrm{e}}(\boldsymbol{r})$ which can be used, in principle, to estimate the characteristic level of magnetic fluctuations $\left\langle B^{\prime 2}\right\rangle / B_{0}^{2}$ and the size of turbulent "cells"; however, this analysis requires the spectra $p(\lambda)$ and $\chi(\lambda)$.

The numerical calculations demonstrate that the observable polarization $(p \geq 0.01 \%)$ corresponds to the values $\delta_{\mathrm{s}} \tau_{\mathrm{env}} \leq$ $10^{3}$. Roughly, this means that $\lambda^{2}(\mu \mathrm{m}) B_{\mathrm{s}}(G) \leq 10^{3}$. The photons with energy $1 \mathrm{eV}$ have the wavelength $1.24 \mu \mathrm{m}$. The photons with energy about (10-100) keV have wavelengths of $\approx 10^{-4} \mu \mathrm{m}$. Thus, the surface magnetic fields $B_{\mathrm{s}} \leq 10^{3} \mathrm{G}$ can produce the observable polarization in visible and infrared intervals of the scattered radiation wavelengths. The magnetic fields $B_{\mathrm{s}} \approx\left(10^{9}-10^{11}\right) \mathrm{G}$ can be detected from the polarimetric $\mathrm{X}$-ray and $\gamma$-ray observations.

The presented theory and the particular calculations can be used to interpret the observed polarimetric data from many cosmic objects possessing the magnetized envelopes and jets (quasars, active galactic nuclei, etc.).

\section{References}

Blaes, O. M. 2003, in Les Houches Session LXXVIII, 2002, Accretion discs, jets and high energy phenomena in astrophysics, ed. V. Beskin, et al. (N.Y.: Springer), 147

Brown, J. C., \& McLean, L. S. 1977, A\&A, 57, 141

Cherepashchuk, A. M. 2001, Usp. Fiz. Nauk, 171, 864 (Phys. Usp., 44, $821(2001))$

Chesneau, O., Wolf, S., \& Domiciano de Souza, A. 2003, A\&A, 410, 375

Dolginov, A. Z., Gnedin, Yu. N., \& Silant'ev, N. A. 1995, Propagation and polarization of radiation in cosmic media (N. Y.: Gordon \& Breach), 126

Dolginov, A. Z., \& Silant'ev, N. A. 1974, Sov. Astron., 18, 289

Gradshteyn, I. S., \& Ryzhik, I. M. 1994, Table of Integrals, Series and Products, 5-th edition (N.Y.: Academic Press), 439

Gnedin, Yu. N., \& Silant'ev, N. A. 1980, SvA Lett., 6, 344

Gnedin, Yu. N., \& Silant'ev, N. A. 1984, Ap\&SS, 102, 375

Gnedin, Yu. N., \& Silant'ev, N. A. 1997, Basic Mechanisms of Light Polarization in Cosmic Media, Astrophys. Space Phys. Rev., vol. 10 (New York: Gordon \& Breach Publ.), 1

Gnedin, Yu. N., \& Silant'ev, N. A. 2002, Astron. Lett., 28, 438

Gnedin, Yu. N., Silant'ev, N. A., Piotrovich, M. Yu., \& Pogodin, M. A. 2005, Astron. Rep., 49, 179

Hartmann, L. 1983, In ed. J. O. Stenflo, Solar and stellar magnetic fields: origins and coronal effects (Dordrecht: Reidel), IAU Symp., 102, 419

Impey, C. D., Lawrence, C. R., \& Tapia, S. 1991, ApJ, 375, 46

Ishwara-Chandra, C. H., Saikia, D. J., Kapahi, V. K., \& McCarty 1998, Preprint No. 6198 (National Center for Radio Astrophysics)

Lamy, H., \& Hutsemekers, D. 2000, A\&AS, 142, 451

Landstreet, J. D. 1992, Astron. Ap. Rev., 4, 35

Leonard, D. C., Filipenko, A. V., Moran, E. C., Barth, A. J., \& Matheson, T. 2000, ApJ, 536, 239

Long, K. S., Chanan, G. A., Ku, W. H.-M., \& Novick, R. 1979, ApJ, 232, L107

Long, K. S., Chanan, G. A., \& Novick, R. 1980, ApJ, 238, 710

Paatz, G., \& Camenzind, M. 1996, A\&A, 308, 77

Pentericci, L., van Reeven, W., Carilli, C. L., et al. 2000, A\&AS, 145, 121

Pogodin, M. A. 1992, Pis'ma v Astr. Zh., 18, 442

Schmidt, G. D., \& Hines, D. C. 1999, ApJ, 512, 125

Silant'ev, N. A. 2005, A\&A, 433, 1117

Udompraset, P. S., Taylor, G. B., \& Roberts, T. J. 1997, ApJ, 483, L9

Wills, B. J., Wills, D., Breger, M., Antonucci, R. R. J., \& Barvainis, R. 1992, ApJ, 398, 454

Young, S., Hough, J. H., Efstathiou, A., et al. 1996, MNRAS, 281, 1206 